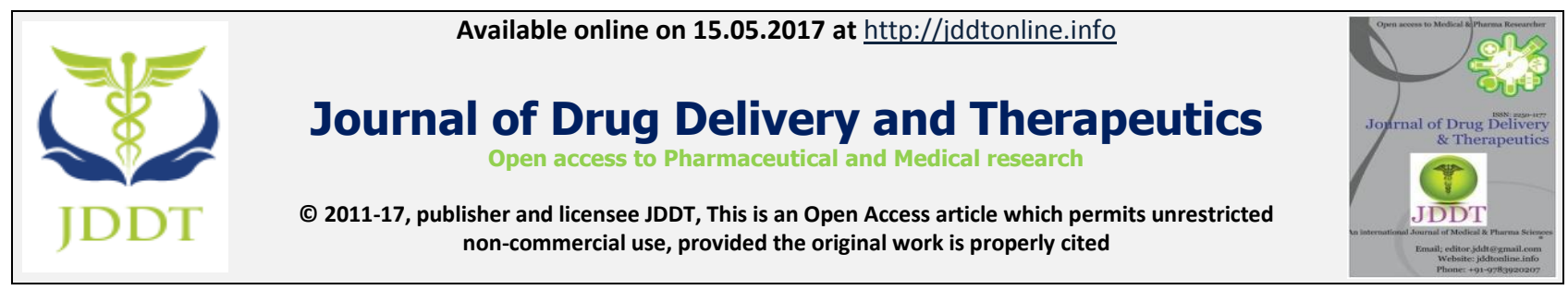

Mini Review

\title{
MULTI-COMPONENT DRUG DELIVERY OF PACLITAXEL
}

\author{
Zhengwei Zhang \\ School of Science, China Pharmaceutical University, Nanjing, Jiangsu, China, 210009
}

Article Info: Received 25 April 2017; Review Completed 07 May 2017; Accepted 07 May 2017, Available online 15 May 2017

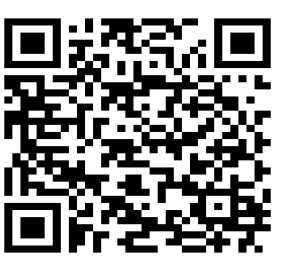
Cite this article as:

Zhang Z, Multi-component drug delivery of Paclitaxel, Journal of Drug Delivery and Therapeutics. 2017; $7(3): 80-83$

DOI: http://dx.doi.org/10.22270/jddt.v7i3.1451

*Address for Correspondence:

Zhengwei Zhang, School of Science, China Pharmaceutical University, Nanjing, Jiangsu, China, 210009, Email: zzwcpu@163.com

\begin{abstract}
Co-delivery systems have been proved to be of much benefit to the anti-cancer drug field. In order to make these drugs successful, nano-particles usage has been promoted. The mini-review has brought forward some of the most prominent co-delivery developments in the field, especially with paclitaxel. The discussion here is never meant to be comprehensive, but to give some new trends and highlights within this area.
\end{abstract}

Keywords: Drug Delivery, Nanomedicine, Co-delivery, Paclitaxel

\section{INTRODUCTION}

Throughout the recent decades, there has occurred an enormous rise in the polymer micelles potential usage as anti-cancer drugs delivery vehicles. ${ }^{1-5}$ These vehicles have definitely offered some significant insight to the way in which treatment can be enforced effectively. ${ }^{6-10}$ This paper has selected various journal articles showing the same and proving to be essential in the field.

\section{Co-delivering paclitaxel and Herceptin}

The article by Lee reports the use of a copolymer which is biodegradable as well as amphiphilic in nature. ${ }^{11}$ The copolymer is used to obtain self-assembled micellar nanoparticles. ${ }^{6,12}$ The copolymer is termed as poly co ammonium bromide sebacate, P (MDS-co-CES). These had the ability to deliver the drugs as well as bio-macro molecules. ${ }^{13}$ These included genes as well as proteins of functional nature either in a simultaneous manner or individual manner within several cell types. ${ }^{14,15}$ Within this article, such nanoparticles were taken into consideration which had cationic micellar nature. These were used as a medium to carry for co-delivering paclitaxel as well as Herceptin. This was done to achieve paclitaxel targeted delivery for receptor 2 growth factor in the epidermis of humans. This was done to suppress toxicity of the cells by activities of synergistic nature. Nanoparticles loaded with Paclitaxel have a size of average nature lesser than $120 \mathrm{~nm}$ and there is a potential of zeta potential for about $60 \mathrm{mV}$. The complexion of Herceptin was done on the nanoparticles surface. ${ }^{16}$ The nano-particle drug loading complication continued to have stability under conditions of physiological simulation with $200 \mathrm{~nm}$ sizes. Herceptin was delivered by nanoparticles in an efficient manner than the bioPorter. This is a protein carrier with lipid base available commercially. The nano-particles with Herceptin depicted an effectiveness of anti-cancer highly. Daily treatment was repeated twice with the element of Herceptin depicting high cell toxicity significantly and particularly within overexpressed cancer cells in the breast in comparison to any individual treatment. Effects of anti-cancer coming from such a co-delivery system were thoroughly investigated within the cancer cells of the human breast. This was completed in alignment with several ranges of the expression level of Her2. These were inclusive of BT474, T47D, and MCF7. The Herceptin co-delivery enhanced the paclitaxel cytotoxicity and such enhancement depicted to be dependent on the expression levels of HER2. This co-delivery system targeted ability, within the article, was shown by images of 
confocal nature. This depicted a higher uptake of cellular nature significantly. This was taken up by the BT474 overexpressed cells of HER2 in comparison to the negative HER3 negative cells of HEK293. This system of co-delivery might have essential implications clinically against the overexpressed cancer cells in the breast.

\section{Co-delivering siRNA and paclitaxel}

Preparation of biodegradable cation micelles was done from the triblock copolymer of PDMAEMA-PCLPDMAEMA and applicable for the siRNA delivery as well as paclitaxel over the cells of cancer. ${ }^{17}$ The copolymers of PDMaEMA-PCL-PDMAEMA were gained readily through the reversed chain transfer of additional fragmentation (also known as RAFT) with dimethylamino ethyl methacrylate polymerization. This was done through the use of CPAD-PCL-CPADN (4cyanopentanoic acid dithionaphthalenoate) as an agent of micro-RAFT. ${ }^{18}$ The PDMAEMA blocks molecular weights under the control of monomer ranged from 2700 to 9100. Such copolymers with triblocking resulted in the formation of water-based nano-sized micelles with positive charges over the surface. These ranged from 29.5 and $35.5 \mathrm{mV}$. A low cytotoxicity was revealed by micelles 1 and 2 . The assay test of gel retardation depicted that first and second micelles could complicate effectively with the use of siRNA as well as over the ratios of N/P for $4 / 2$ and $2 / 1$. The GFP siRNA notably having complexed with the 1 st micelle depicted enhanced significance for gene silencing. ${ }^{19-21}$ It depicted more efficiency in comparison to the $20 \mathrm{kDa}$ PDMAEMA formulation. 1st micelle, moreover, loaded with the enzyme of paclitaxel depicted higher efficacy for drug compared with PC2 cells with free paclitaxel. This is because of most likeliness for cellular uptake improvement. The VEGF siRNA combined delivery, as well as paclitaxel, depicted a VEGF expression efficient knockdown. Studies using confocal laser scanning microscope over GFP siRNA complexed with micelle having nile red loading depicted that nile red could be delivered within the MDA-MB-435-GFP. This was expressed by GFP cells. Such expression of GFP was inhibited significantly. Such results showed that biodegraded micelles with cations have more promise for the siRNA and lipophile combined delivery for anticancer drugs.

\section{Co-delivering doxorubicin and paclitaxel}

The research by Wang et al. stated that using the single drug for chemotherapy has shown certain anti-tumor treatment limitations. ${ }^{22}$ When combining the drugs, it results in better performance. In this article, the authors proposed minimizing each drug's amount. This was done to gain the effect of synergistic nature for therapies of cancer. Various researchers have attempted to deliver drugs with chemotherapeutics in a simultaneous manner through the use of carriers of drugs such as liposomes, micelles, and nanoparticles of inorganic nature. The article reported nanoparticles with core shells to be emulsified in a double manner from copolymers having amphiphilic nature. The copolymer used by them was methoxy poly (ethylene glycol)-poly (lactide-co- glycolide). Such nanoparticles resulted in offering benefits over related nano-carriers because they had the ability of easy fabrication. This was done through an improvised method for double emulsion known as biocompatibility. It showed higher efficacy for loading. More significantly, these nanoparticles had the ability of co-delivering doxorubicin of hydrophilic nature as well as paclitaxel of hydrophobic nature. ${ }^{23,24}$ The nanoparticles loaded with the drug, had a good ability to polydisperse. This depicted that they were subjected more readily to controlled distribution of size. Drug release studies, as well as co-delivery systems cellular uptake, depicted that the drugs were taken up effectively through cells and simultaneously released.

\section{Co-delivering tariquidar and paclitaxel}

The study by Patel et al. proposed that p-gp inhibitors with higher efficacious ability are to be used to show success to overcome MDR. ${ }^{25}$ Such inhibitor is taken in the article as tariquidar. ${ }^{26} \mathrm{P}$-gp, however, can further be expressed within general tissues such as the barriers in the blood brain, track of gastrointestinal nature, spleen as well as kidney. For maximizing the P-gp inhibitor efficacy as well as reducing the toxicity systemically, it becomes essential to consider limiting the P-gp inhibitors exposure as well as the drugs of anti-cancer for normal tissues. This further results in increasing the tumor cells co-localization. Within the article, the authors indulged in investigating the P-gp inhibitor codelivery and drug of cytotoxic nature, namely paclitaxel within the cells of the tumor for reversing the MDR (multi-drug resistance) through the use of liposomes with long circulation. These liposomes along with tariquidar depicted essential resistant variant resensitization for the paclitaxel-resistant variance. This could have a correlation with enhanced paclitaxel accumulation within the cells of the tumor.

\section{Co-delivering paclitaxel and siRNA with lipid nanoparticle}

According to the discovery by $\mathrm{Yu}$ et al., nano-particles with cationic solids of lipids were formulated for paclitaxel co-delivery with siRNA. ${ }^{27}$ Methods of emulsification solidification were used for preparing csiN based on 1.2-Dioleoyl-sn-glycero3ethylphosphochonline. ${ }^{28-30}$ Zeta potential was used for characterizing the cSLN loaded with PTX as well as retardation of gels with small interfering RNA complexes. The PcSLN sizes were not essentially different from the empty cSLN. The cSLN utilization enhanced the uptake at the cellular level of dsRNA fluorescence within the carcinoma KB cells. These cells are present in the epithelium of humans. This is further combined with PcSLN complexing it to dsRNA labeled through fluorescence. This results in promotion of maximum uptake. For therapeutic siRNA co-delivery, siRNA specific MCL1 for humans was complexed around PcSLN. The control in this situation was the complexion of siRNA specific to luciferase over PcSLN. The levels of MC1 mRNA were reduced in an essential manner within the cells of $\mathrm{KB}$ undergoing treatment with the complexion of siMCL over PcSLN. Collectively, the article demonstrated the cSLN 
potential for developing various lipophile based drugs for anti-cancer co-delivery systems as well as siRNAs therapeutically.

\section{CONCLUSION}

This is to conclude that there have been several developments in the cancer treatment field. However, multi-drug resistant has remained a concurring problem. ${ }^{31-34}$ All the articles being discussed in this

\section{REFERENCES}

1. Dai W, Wang X, Song G, Liu T, He B, Zhang H, et al. Combination antitumor therapy with targeted dualnanomedicines. Advanced Drug Delivery Reviews. 2017,

2. Kraft J C, Freeling J P, Wang Z, and Ho R J. Emerging research and clinical development trends of liposome and lipid nanoparticle drug delivery systems. Journal of pharmaceutical sciences. 2014, 103: 29-52

3. Mussi S V, Silva R C, de Oliveira M C, Lucci C M, de Azevedo R B, and Ferreira L A M. New approach to improve encapsulation and antitumor activity of doxorubicin loaded in solid lipid nanoparticles. European Journal of Pharmaceutical Sciences. 2013, 48: 282-290

4. Liu F, Sun Y, Kang C, and Zhu H. Pegylated Drug Delivery Systems: From Design to Biomedical Applications. Nano LIFE. 2016, 6: 1642002

5. Qiao H, Fang D, Chen J, Sun Y, Kang C, Di L, et al. Orally delivered polycurcumin responsive to bacterial reduction for targeted therapy of inflammatory bowel disease. Drug Delivery. 2017, 24: 233-242

6. Wan C, Allen T, and Cullis P. Lipid nanoparticle delivery systems for siRNA-based therapeutics. Drug delivery and translational research. 2014, 4: 74-83

7. Kang C, Sun Y, Wang M, and Cheng X. Nanosized camptothecin conjugates for single and combined drug delivery. European Journal of BioMedical Research. 2016, 2: 8-14

8. Kang C, Sun Y, Zhu J, Li W, Zhang A, Kuang T, et al. Delivery of Nanoparticles for Treatment of Brain Tumor. Current Drug Metabolism. 2016, 17: 745-754

9. Sun Y, Kang C, Zhang A, Liu F, Hu J, Zhong X, et al. Codelivery of dual-drugs with nanoparticle to overcome multidrug resistance. European Journal of BioMedical Research. 2016, 2: 12-18

10. Cheng X. Developing organic and inorganic nanomedicine for cancer therapy. Journal of Drug Delivery and Therapeutics. 2017; 7(3):1-4. DOI: 10.22270/jddt.v7i2.1367

11. Lee A L, Wang Y, Cheng H Y, Pervaiz S, and Yang Y Y. The co-delivery of paclitaxel and Herceptin using cationic micellar nanoparticles. Biomaterials. 2009, 30: 919-927

12. Zhang L, Yang M, Wang Q, Li Y, Guo R, Jiang X, et al. 10Hydroxycamptothecin loaded nanoparticles: preparation and antitumor activity in mice. Journal of controlled release. 2007 , 119: $153-162$

13. Cheng $X$, and Lee $R$ J. The role of helper lipids in lipid nanoparticles (LNPs) designed for oligonucleotide delivery. Adv Drug Deliv Rev. 2016, 99: 129-137; doi: 10.1016/j.addr.2016.01.022.

14. Chen Y, Bian Y, Sun Y, Kang C, Yu S, Fu T, et al. Identification of 4-aminoquinoline core for the design of new cholinesterase inhibitors. PeerJ. 2016, 4: e2140

15. Han R, Sun Y, Kang C, Sun H, and Wei W. Amphiphilic dendritic nanomicelle-mediated co-delivery of 5-fluorouracil and doxorubicin for enhanced therapeutic efficacy. Journal of Drug Targeting. 2017, 25: 140-148

16. Kang C, Qin J, Osei W, and $\mathrm{Hu}$ K. Regulation of protein kinase C-epsilon and its age-dependence. Biochemical and Biophysical Research Communications. 2017, 482: 1201-1206

17. Zhu C, Jung S, Luo S, Meng F, Zhu X, Park T G, et al. Codelivery of siRNA and paclitaxel into cancer cells by paper comprise of solutions for co-delivering drugs to help in efficiency of anti-cancer drugs. ${ }^{35-37}$ It can be concluded that synergistically combining 2 or more than 2 drugs is a strategy of promising nature.

\section{Acknowledgements}

This work was supported by grants from National Science and Technology Major Projects for "Major New Drugs Innovation and Development"(2013ZX09402203)

biodegradable cationic micelles based on PDMAEMA-PCLPDMAEMA triblock copolymers. Biomaterials. 2010, 31: 2408-2416

18. Song L, Kang C, Sun Y, Huang W, Liu W, and Qian Z. Crocetin Inhibits Lipopolysaccharide-Induced Inflammatory Response in Human Umbilical Vein Endothelial Cells. Cellular Physiology and Biochemistry. 2016, 40: 443-452

19. Cheng X, Liu Q, Li H, Kang C, Liu Y, Guo T, et al. Lipid Nanoparticles Loaded with an Antisense Oligonucleotide Gapmer Against Bcl-2 for Treatment of Lung Cancer. Pharm Res. 2017, 34: 310-320; doi: 10.1007/s11095-016-2063-5.

20. Duan Y, Pei K, Cai H, Tu S, Cheng X, Zhang Z, et al. Strategy of integrated evaluation on treatment of traditional Chinese medicine as 'interaction of system to system'and establishment of novel fuzzy target contribution recognition with herb-pairs, a case study on Astragali Radix-Fructus Corni. Molecular and Cellular Endocrinology. 2016, 434: 219-237

21. Duan Y, Pei K, Cai H, Tu S, Zhang Z, Cheng X, et al. Bioactivity evaluation-based ultra high-performance liquid chromatography coupled with electrospray ionization tandem quadrupole-time-of-flight mass spectrometry and novel distinction of multi-subchemome compatibility recognition strategy with Astragali Radix-Fructus Corni herb-pair as a case study. Journal of pharmaceutical and biomedical analysis. 2016, 129: 514-534

22. Wang $\mathrm{H}$, Zhao $\mathrm{Y}$, Wu Y, Hu Y-1, Nan K, Nie G, et al. Enhanced anti-tumor efficacy by co-delivery of doxorubicin and paclitaxel with amphiphilic methoxy PEG-PLGA copolymer nanoparticles. Biomaterials. 2011, 32: 8281-8290

23. Li H, Cheng X, Liu Y, Lee Y B, Kim D J, Ahn C-h, et al. Folate receptor-targeted lipid coated albumin nanoparticles (FLCAN) for therapeutic delivery of RX-0201 (Archexin®), an antisense oligonucleotide against Akt-1. 2016,

24. Li H, Quan J, Zhang M, Yung B C, Cheng X, Liu Y, et al. Lipid-Albumin Nanoparticles (LAN) for Therapeutic Delivery of Antisense Oligonucleotide against HIF-1alpha. Mol Pharm. 2016, 13: 2555-2562; doi: 10.1021/acs.molpharmaceut.6b00363.

25. Patel N R, Rathi A, Mongayt D, and Torchilin V P. Reversal of multidrug resistance by co-delivery of tariquidar (XR9576) and paclitaxel using long-circulating liposomes. International journal of pharmaceutics. 2011, 416: 296-299

26. XU S-h, Chen K, CHEN M-1, ZHOU P-p, HE G-w, CUI Y-j, et al. Dynamic expression of AQP4 in early stageof ischemia/reperfusion rats and cerebral edema. Chinese Pharmacological Bulletin. 2016: 1433-1441

27. Yu Y H, Kim E, Park D E, Shim G, Lee S, Kim Y B, et al. Cationic solid lipid nanoparticles for co-delivery of paclitaxel and siRNA. European Journal of Pharmaceutics and Biopharmaceutics. 2012, 80: 268-273

28. Waller A P, George M, Kalyanasundaram A, Kang C, Periasamy $\mathrm{M}, \mathrm{Hu} \mathrm{K}$, et al. GLUT12 functions as a basal and insulin-independent glucose transporter in the heart. Biochimica et Biophysica Acta (BBA)-Molecular Basis of Disease. 2013, 1832: 121-127

29. Guo X K, Sun H P, Shen S, Sun Y, Xie F L, Tao L, et al. Synthesis and evaluation of gambogic acid derivatives as 
antitumor agents. Part III. Chemistry \& biodiversity. 2013, 10: 73-85

30. Sun H, Zhu J, Chen Y, Sun Y, Zhi H, Li H, et al. Docking Study and Three-Dimensional Quantitative Structure-Activity Relationship (3D-QSAR) Analyses and Novel Molecular Design of a Series of 4-Aminoquinazolines as Inhibitors of Aurora B Kinase. Chinese Journal of Chemistry. 2011, 29: 1785-1799

31. Xue X, Zhao N-Y, Yu H-T, Sun Y, Kang C, Huang Q-B, et al. Discovery of novel inhibitors disrupting HIF-1 $\alpha$ /von HippelLindau interaction through shape-based screening and cascade docking. PeerJ. 2016, 4: e2757

32. Yang Z, Xie J, Zhu J, Kang C, Chiang C, Wang X, et al. Functional exosome-mimic for delivery of siRNA to cancer: in vitro and in vivo evaluation. Journal of Controlled Release. 2016, 243: 160-171

33. Yao Z, Sun Y, and Kang C. Structure and Self-Assembly of Multicolored Naphthalene Diimides Semiconductor. Nano LIFE. 2016, 6: 1642007

34. Zhong X, Sun Y, Kang C, and Wan G. The theory of dielectrophoresis and its applications on medical and materials research. European Journal of BioMedical Research. 2017, 2: 7-11

35. Sun Y, and Kang C. Self-Assembly of Peptides into Hydrogel. Journal of Organic \& Inorganic Chemistry. 2016,

36. Sun Y, Kang C, Liu F, and Song L. Delivery of Antipsychotics with Nanoparticles. Drug Development Research. 2016, 77: 393-399

37. Sun Y, Kang C, Yao Z, Liu F, and Zhou Y. Peptide-Based Ligand for Active Delivery of Liposomal Doxorubicin. Nano LIFE. 2016, 6: 1642004 